\title{
Investigation of Genipin Cross-Linked Microcapsule for Oral Delivery of Live Bacterial Cells and Other Biotherapeutics: Preparation and In Vitro Analysis in Simulated Human Gastrointestinal Model
}

\author{
Hongmei Chen, Wei Ouyang, Christopher Martoni, Fatemeh Afkhami, Bisi Lawuyi, \\ Trisna Lim, and Satya Prakash
}

Department of Biomedical Engineering and Physiology, Biomedical Technology and Cell Therapy Research Laboratory, Artificial Cells and Organs Research Centre, Faculty of Medicine, McGill University, Montreal, Qc, Canada H3A 2B4

Correspondence should be addressed to Satya Prakash, satya.prakash@mcgill.ca

Received 15 March 2010; Accepted 31 May 2010

Academic Editor: Shanfeng Wang

Copyright (C) 2010 Hongmei Chen et al. This is an open access article distributed under the Creative Commons Attribution License, which permits unrestricted use, distribution, and reproduction in any medium, provided the original work is properly cited.

\begin{abstract}
Oral therapy utilizing engineered microorganisms has shown promise in the treatment of many diseases. By microencapsulation, viable cells can overcome the harsh gastrointestinal (GI) environment and secrete needed therapeutics into the gut. These engineered cells should be encased without escaping into the GI tract for safety concerns, thus robust microcapsule membrane is requisite. This paper examined the GI performance of a novel microcapsule membrane using a dynamic simulated human GI model. Results showed that the genipin cross-linked alginate-chitosan (GCAC) microcapsules possessed strong resistance to structural disintegration in the simulated GI environment. Leakage of encapsulated high molecular weight dextran, a model material to be protected during the simulated GI transit, was negligible over $72 \mathrm{~h}$ of exposure, in contrast to considerable leakage of dextran from the non-cross-linked counterparts. These microcapsules did not alter the microflora and enzymatic activities in the simulated human colonic media. This study suggested the potential of the GCAC microcapsules for oral delivery of live microorganisms and other biotherapeutics.
\end{abstract}

\section{Introduction}

Advances in molecular biology research have introduced a wide range of genetically engineered (GE) microorganisms with a superior capacity to produce disease-modifying substrates, such as cytokines, enzymes, vaccines, hormones, antibodies, growth factors, and other therapeutic products $[1,2]$. The use of these microorganisms opens up new hopes of treating a wide array of human diseases. Because the secreted biologics are generally fragile and easily degraded or denatured [3], encapsulation technology may offer significant advantages over the conventional biotechnological production methods. Being protected against external stresses, encapsulated bacteria remain viable and functional. They can be delivered proximally to the target site in vivo and continuously secrete therapeutic products to the host at a more effective concentration [4]. Recent research on the microencapsulation of GE cells has demonstrated great potential in the treatment of kidney failure, cancers, hypercholesteraemia, and many other diseases [5-13].

Oral ingestion is usually a preferred route of administration for therapy; however, microcapsules containing bacterial cells and other biotherapeutic molecules can be disrupted in the harsh gastrointestinal (GI) system by a number of means such as low $\mathrm{pH}$, antimicrobial substances and mechanical stress [14]. Furthermore, the eruption of microcapsules and the subsequent release of engineered bacteria could induce many adverse effects on the body [2]. It was previously shown that oral administration of repeated doses of bacteria may stimulate a host immune response $[15,16]$. Propagation of foreign bacteria in the GI tract may cause uncontrolled and persistent production of harmful substances, and may 
detrimentally disrupt and/or replace the natural microflora $[2,17]$. There are also risks of immunomodulation and gene transfer associated with the use of novel microorganisms [18]. Therefore, it is essential that GE bacteria be encased in the microcapsules, perform the therapeutic functions during the GI transit, and be excreted along with the intact microcapsules in feces without being retained in the body, even though these GE cells are classified as nonpathogenic [7]. To fulfill these requirements, it is important to maintain the structural integrity of microcapsules and thus prevent cell leakage during the GI transit. Current literature reports on oral delivery systems mainly focus on the controlled release of encapsulated contents, for example, probiotics and drugs $[4,19-23]$. Scanty research is available on microcapsules intended to retain cells throughout the GI transit.

Alginate and chitosan are biomaterials widely studied for cell encapsulation because of their excellent biocompatibility, status as FDA approved food additives, and mild process conditions [24-28]. It was reported that the ionically linked alginate-chitosan (AC) membrane improved gastric survival of probiotics, but some limitations such as inadequate stability, susceptibility to degradation and cell leakage persist [29-33]. Genipin and its derivatives, extracted from gardenia fruits [34], have traditionally been used as a herbal medicine and a natural colorant in the food industry [35]. Previous research has demonstrated its low cytotoxicity and potential in protein and live cell delivery [36-38]. We have previously developed a novel covalently cross-linked microcapsule system composed of a calcium alginate core with a genipin cross-linked chitosan membrane $[36,39]$. Our recent data showed that this microcapsule membrane possessed strong membrane stability and resistance to enzymatic degradation [40]. The purpose of this study is to further evaluate the potential of this genipin cross-linked alginate-chitosan (GCAC) microcapsule system for GI applications by using a dynamic human GI model. For comparison, the frequently used AC microcapsules were also tested.

\section{Materials and Methods}

2.1. Chemicals. Sodium alginate (low viscosity), and fluorescein isothiocyanate (FITC) labeled dextran $(M w 2,000$ KD) were supplied by Sigma-Aldrich, USA. Chitosan (low viscosity, $M v=7.2 \times 10^{4}$, degree of deacetylation or $\mathrm{DDA}=73.5 \%)$ and genipin were purchased from Wako BioProducts, USA. 4-nitrophenyl- $\alpha$-D galactopyranoside, 4nitrophenyl- $\alpha-\mathrm{D}$ glucopyranoside, and 4-nitrophenyl- $\beta-\mathrm{D}$ galactopyranoside were obtained from Acros Organics, USA. 4-nitrophenol, 4-nitrophenyl- $\beta$-D glucopyranoside, and 4nitrophenyl- $\beta$-D glucuronide were purchased from SigmaAldrich, USA. All other reagents and solvents were of reagent grade and used as received without further purification.

2.2. Preparation of Microcapsules. The AC and GCAC microcapsules were prepared as per previously reported protocol [39]. Unless otherwise specified, the cross-linking reaction was performed by suspending the AC microcapsules in a genipin solution $(2.5 \mathrm{mg} / \mathrm{mL})$ at room temperature (RT). Sterile microcapsules were prepared similarly except that the entire encapsulation procedure was carried out in a biological containment hood and all solutions used were either $0.22 \mu \mathrm{m}$ filtered or autoclaved to ensure sterility. Microcapsules containing high molecular weight FITClabeled dextran were prepared by mixing FITC-dextran with an alginate solution, making the final concentrations of alginate and FITC-dextran at $15 \mathrm{mg} / \mathrm{mL}$ and $2 \mathrm{mg} / \mathrm{mL}$, respectively. The subsequent processes, including the formation of alginate beads, coating and cross-linking were performed using the aforementioned procedures.

\subsection{Simulation of the Human Gastrointestinal (GI) Environ-} ment. The human GI conditions used in this study were simulated in vitro by means of five sequential bioreactors (Figure 1). Each compartment simulates a different part of the human GI tract: the stomach, the small intestine, the ascending colon, the transverse colon, and the descending colon $[41,44]$. Human fecal slurries containing normal human GI bacterial cells were inoculated into the simulated colon (the last three vessels). Food content of human western diet suspension, composed of (per liter) $1 \mathrm{~g}$ arabinogalactan, $2 \mathrm{~g}$ pectin, $1 \mathrm{~g}$ xylan, $3 \mathrm{~g}$ potato starch, $0.4 \mathrm{~g}$ glucose, $3 \mathrm{~g}$ yeast extract, $1 \mathrm{~g}$ peptone, $4 \mathrm{~g}$ mucin, and $0.5 \mathrm{~g}$ cystein, was fed to the first vessel three times a day. After feeding, acidification of the stomach $(\mathrm{pH} \leq 2)$ occurred, followed by neutralization $(\mathrm{pH} \geq 6.8)$ in the second vessel and addition of simulated pancreatic juice $(0.9 \mathrm{~g}$ pancreatin, $6 \mathrm{~g}$ bile salts, and $12 \mathrm{~g} \mathrm{NaHCO}_{3}$ per liter) to the simulated small intestine. Afterwards, the suspension was transferred to the simulated ascending colon, the transverse colon, and the descending colon, and finally excreted as effluent. The first two reactors were of the fill-and-draw principle with programmed periods of residence and stirring, and the last three were continuously agitated (approximately $250 \mathrm{rpm}$ ). The whole system was maintained under anaerobic conditions by flushing the headspace of each vessel with N2 for 15 minutes every day and the temperature of each vessel was kept constant at $37^{\circ} \mathrm{C}$ by a thermostat. The $\mathrm{pH}$ conditions, fluid volume, retention time at each stage, as well as the entire transit were simulated under computer control. This in vitro human GI model was validated against in vivo data by earlier studies [41].

\subsection{Resistance of Microcapsules to the Simulated Human} GI Transit. To study the microcapsule resistance to the simulated human GI transit, microcapsules $(0.80 \mathrm{~g})$ were exposed to the simulated human GI fluids for the estimated maximum period of time for the human GI transit (Table 1) [42]. Microcapsule samples were withdrawn at varied stages for morphological examination under an inverted microscope (LOMO, PC), and microphotographs taken as records using a digital camera (Canon Power shot G2, Japan). Percent defective microcapsules were estimated in three randomly picked observation fields.

To assess the recovery of microcapsules after the simulated human GI transit, microcapsules of known weight were placed in a sealed teabag-like container and exposed to the simulated GI media according to the timetable described in Table 1. At the final stage, the retrieved microcapsules were washed, dried using filter paper for approximately $10 \mathrm{~min}$ 


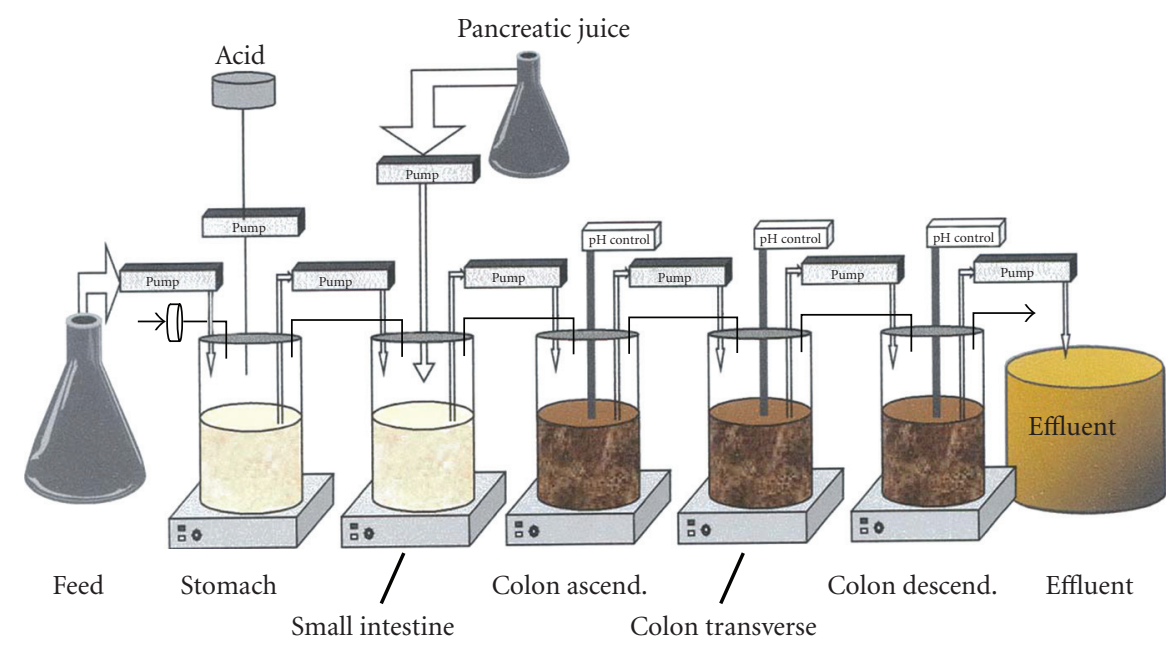

(a)

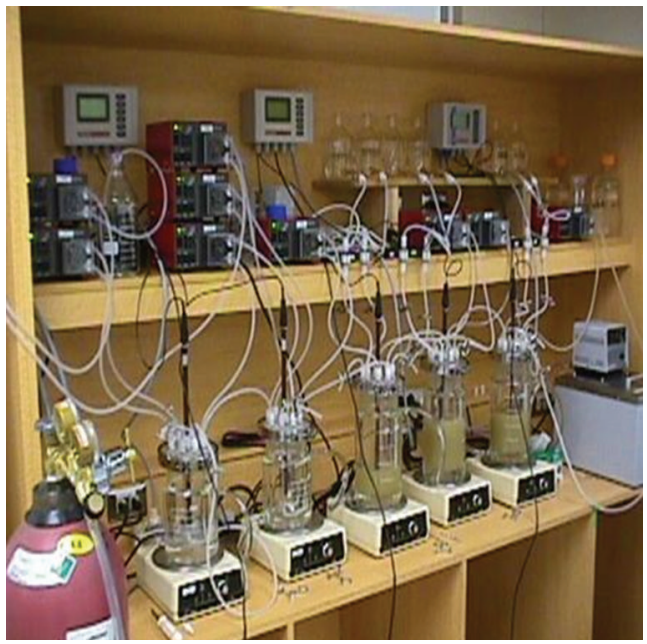

(b)

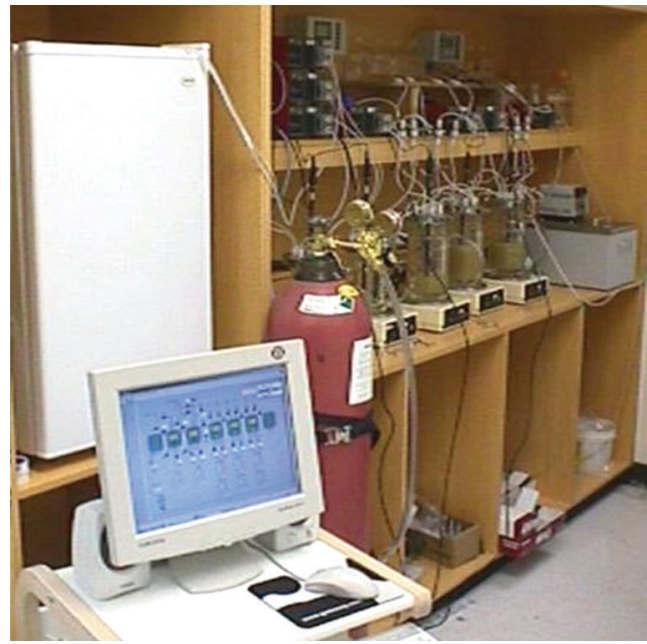

(c)

FIgURE 1: Computer controlled dynamic in vitro human GI model. (a) schematic; (b) 5-bioreactors set-up; and (c) online computer control.

and weighed. Percent recovery was defined as: \% recovery $=\left(W_{0}-W\right) / W_{0} * 100$, where $W_{0}$ and $W$ are the weights of the microcapsules before and after being exposed to the simulated GI transit, respectively.

2.5. Retention of Encapsulated Macromolecules against Leaching to the Simulated Human GI Fluids. To evaluate the capacity of the GCAC microcapsules to retain enclosed large-sized materials, high molecular weight FITC-dextran was encapsulated as a model macromolecule. These FITC-dextrancontaining microcapsules $(0.60 \pm 0.01 \mathrm{~g})$ were exposed to $2 \mathrm{~mL}$ of the simulated human gastric fluid taken from the GI model and incubated in an Environ shaker at $37^{\circ} \mathrm{C}, 100 \mathrm{rpm}$ for 1 hour, followed by a 72 hours incubation in $2 \mathrm{~mL}$ of the simulated human intestinal fluid from the GI model $\left(37^{\circ} \mathrm{C}\right.$, $100 \mathrm{rpm})$. Samples of the incubation media were withdrawn periodically and leaking of the encapsulated FITC-dextran was assessed spectrofluorometrically using a Microplate Fluorescence Reader (FLx800, Bio-Tek Instruments, Inc.) at absorption and emission wavelengths of $485 \mathrm{~nm}$ and $528 \mathrm{~nm}$, respectively. Volume of the incubation medium was kept constant by adding fresh simulated fluid after sampling. Data are presented as mean \pm s.d. from triplicate experiments.

2.6. Effect of Microcapsules on the Simulated Gut Microflora. To investigate the influence of oral administered microcapsules on gut microflora, sterile microcapsules $(1.0 \mathrm{~g})$ were mixed with the suspension of the simulated transverse colon $(10 \mathrm{~mL})$. After $0,6,12$, and 24 hours of anaerobic incubation at $37^{\circ} \mathrm{C}$, samples of the incubation medium were aseptically withdrawn and serially diluted with physiological saline. Bacterial enumeration for specific fecal marker microorganisms, Escherichia coli, Staphylococcus sp., Lactobacillus sp. as well as total aerobes and total anaerobes, was performed using an agar-plate-count assay. The plating media and incubation conditions used in the experiments are listed in Table 2. The simulated colonic suspension without microcapsules was used as control. 
TABLE 1: Exposure of microcapsules to the simulated human GI transit (72 $\mathrm{h}$ in total) and the corresponding morphological changes.

\begin{tabular}{|c|c|c|c|c|c|}
\hline $\begin{array}{l}\text { Compartment } \\
\text { in GI model }\end{array}$ & $\begin{array}{l}\text { Stomach } \\
\left(\mathrm{V} 1^{a}\right)\end{array}$ & $\begin{array}{l}\text { Small intestine } \\
\qquad\left(\mathrm{V} 2^{a}\right)\end{array}$ & $\begin{array}{l}\text { Ascend. Colon } \\
\left(\mathrm{V} 3^{a}\right)\end{array}$ & $\begin{array}{c}\text { Transverse colon } \\
\qquad\left(\mathrm{V} 4^{a}\right)\end{array}$ & $\begin{array}{c}\text { Descend. Colon } \\
\left(\mathrm{V} 5^{a}\right)\end{array}$ \\
\hline $\mathrm{pH}$ & $\leq 2$ & $7.2-7.4$ & $5.6-5.8$ & $6.2-6.4$ & $6.6-6.8$ \\
\hline $\begin{array}{l}\text { Exposure time } \\
\text { (h) }\end{array}$ & 2 & 4 & 18 & 24 & 24 \\
\hline $\begin{array}{l}\text { Microcapsule } \\
\text { morphology }^{b}\end{array}$ & Intact & $\begin{array}{l}\text { Swelled, } \sim 15 \% \\
\quad \text { deformed }\end{array}$ & Collapsed & $\begin{array}{c}\text { Collapsed or } \\
\text { dissolved }\end{array}$ & $\begin{array}{l}\text { Adhesive, } \sim 30 \% \\
\text { ruptured }\end{array}$ \\
\hline $\begin{array}{l}\text { Microcapsule } \\
\text { morphology }\end{array}$ & Intact & $\begin{array}{c}\text { Slightly } \\
\text { swelled, }<1 \% \\
\text { burst }\end{array}$ & $\begin{array}{l}\text { Spherical, }<2 \% \\
\text { broken }\end{array}$ & Spherical intact & Spherical intact \\
\hline
\end{tabular}

${ }^{a}$ Vessel (V) of bioreactors in the GI model representing the human GI tract.

${ }^{b}$ Microcapsules cross-linked by genipin at $4^{\circ} \mathrm{C}$.

${ }^{c}$ Microcapsules cross-linked by genipin at RT.

TABLE 2: Media and incubation conditions used for enumeration of representative microbes in the simulated human colon.

\begin{tabular}{lccc}
\hline Microbial group & Medium & Incubation conditions and time & Colonies formed \\
\hline Total aerobes & Brain heart infusion agar & Aerobic, $37^{\circ} \mathrm{C}, 24$ hours & White \\
Total anaerobes & Brain heart infusion agar & Anaerobic, $37^{\circ} \mathrm{C}, 72$ hours & White \\
Escherichia coli & Mc Conkey agar & Aerobic, $43^{\circ} \mathrm{C}, 24$ hours & Red-purple \\
Staphylococcus sp. & Mannitol Salt agar & Aerobic, $37^{\circ} \mathrm{C}, 48$ hours & White with yellow/purple zone \\
Lactobacillus sp. & Rogosa agar & Anaerobic, $37^{\circ} \mathrm{C}, 72$ hours & White \\
\hline
\end{tabular}

\subsection{Effect of Microcapsules on the Microbial Enzyme Activities} in the Simulated Colonic Media. To assess the effect of oral administered microcapsules on the GI microbial enzyme activities, the suspension from the simulated transverse colon $(20 \mathrm{~mL})$ was incubated anaerobically at $37^{\circ} \mathrm{C}$ in the presence of sterile microcapsules $(2.0 \mathrm{~g})$ for up to 24 hours. At different time points of 0,12 , and 24 hours, the enzymatic activities of $\beta$-galactosidase, $\beta$-glucosidase, $\beta$-glucuronidase, $\alpha$ galactosidase, and $\alpha$-glucosidase in the incubation medium were analyzed spectrometrically using the method described earlier $[43,44]$. The absorbance at $405 \mathrm{~nm}$ was recorded by a $\mu \mathrm{Quant}$ multiplate reader (Bio-Tek Instruments). The simulated colonic fluid free of microcapsules was used as control. Results are expressed as percentage of enzymatic activities relative to the control at each time point. Data of the control samples at each time point were normalized to $100 \%$.

Numerical values are shown as mean \pm standard deviation. Statistical analyses used the two-tailed Student's $t$-test with $P<.05$ considered significantly different.

\section{Results}

3.1. Resistance of Microcapsule to the Simulated Human GI Transit. To assess the resistance to the GI environment, the GCAC microcapsules were exposed to the simulated GI media representing different phases of digestion for a length of time based on the estimated maximum retention in the human GI tract (Table 1). Figure 2 depicts the photomicrographs of the microcapsules after exposure to the simulated human GI fluids. The tested microcapsules remained morphologically stable during the simulated gastric incubation (2 hours, $\mathrm{pH} \leq 2.0$ ) (Figures $2(\mathrm{a}), 2(\mathrm{~d})$ ), but behaved differently in the subsequent simulated intestinal transit depending on the extent of cross-linking. The GCAC microcapsules cross-linked at $4^{\circ} \mathrm{C}$ swelled appreciably in the simulated small intestine ( $\mathrm{pH} 7.2-7.4)$ (Figure 2(b)). They became fragile and adhesive in the simulated descending colon where shriveled or partially dissolved beads were observed. When leaving the simulated descending colon, $30-40 \%$ of the microcapsules lost their structural integrity (Figure 2(c)). In contrast, the majority of the GCAC microcapsules cross-linked at higher temperature (RT) maintained their physical stability during the entire simulated human GI transit (Figures 2(e) and 2(f)), with more than $80 \%$ recovery after 72 hours exposure (Figure 3 ). In comparison, the recovery of the GCAC microcapsules with less cross-link (cross-linked at $4^{\circ} \mathrm{C}$ ) was lower $(61.4 \%)$ (Figure 3).

3.2. Retention of Encapsulated HMW Dextran during the Simulated GI Transit. To examine the capacity of the GCAC microcapsules to encase large-sized materials in the GI environment, encapsulated high molecular weight FITC-labeled dextran was exposed to the simulated human GI media. Being a large polymer of $2,000 \mathrm{KD}$, this fluorescent probe was indefinitely withheld inside the intact microcapsules and could not leak out unless the microcapsule membranes became defective or damaged [45]. During the first hour of exposure to the simulated gastric fluid, no FITC-dextran was detected in the incubation medium (data not shown). In the subsequent exposure to the simulated intestinal medium, the leakage of the encapsulated FITC-dextran from the noncross-linked AC microcapsules increased gradually with the incubation time, attaining the fluorescence intensity of 70 , 100 and 115 at 24, 48 and 72 hours, respectively. In contrast, 


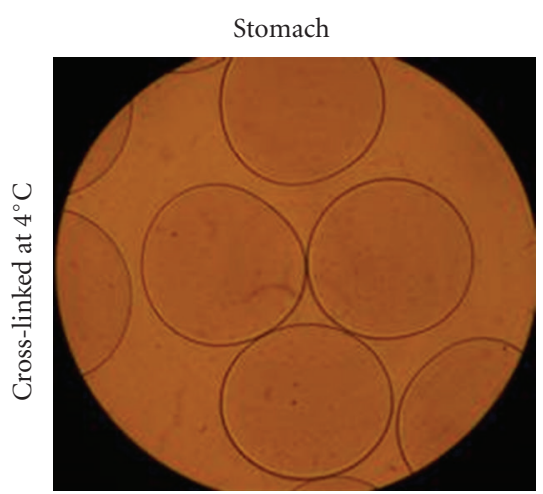

(a)

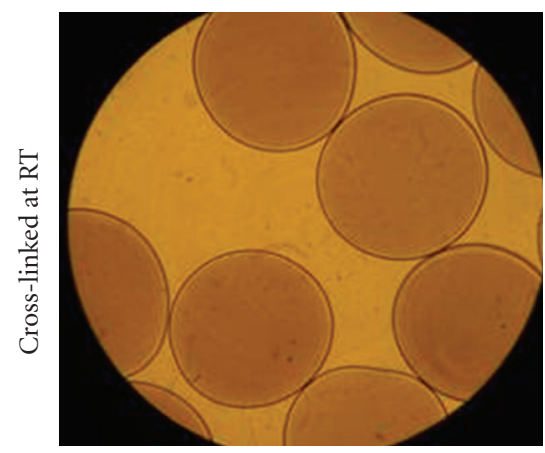

(d)

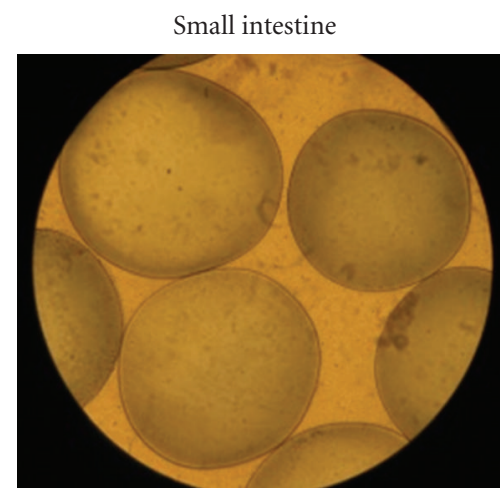

(b)

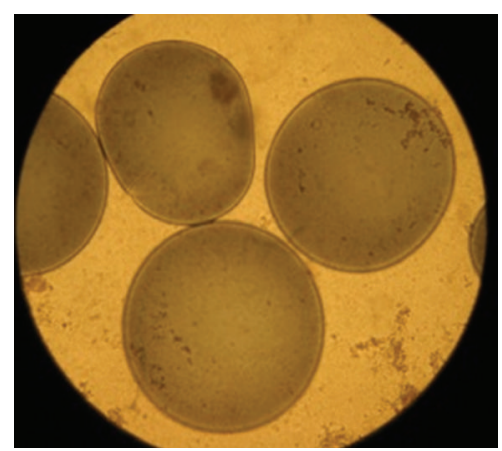

(e)

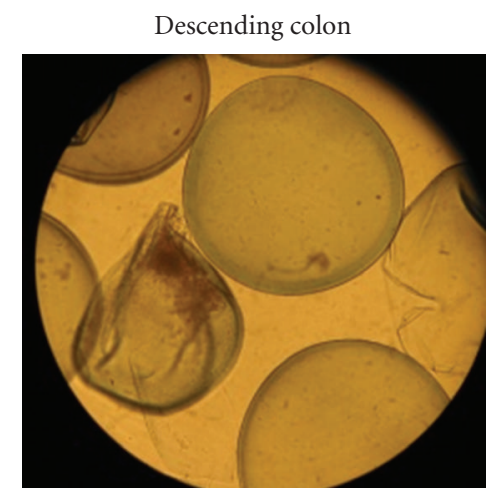

(c)

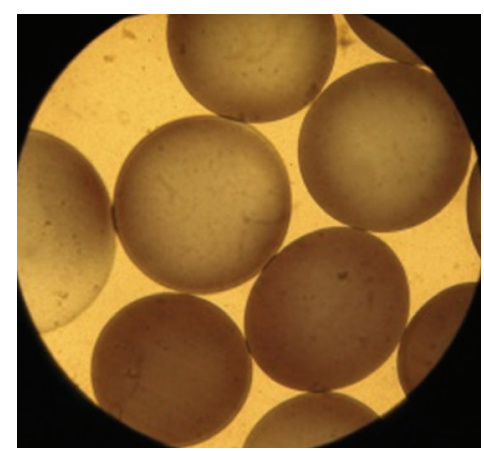

(f)

FIGURE 2: Microphotographs of the GCAC microcapsules cross-linked at $4^{\circ} \mathrm{C}$ (a)-(c) and RT (d)-(f) during the simulated human GI transit in the simulated stomach, small intestine, and descending colons. Original magnification at 90x.

negligible amounts of FITC-dextran escaped from the GCAC microcapsules, with very low fluorescence intensity of the medium detected (not exceeding 50) throughout the experiment (Figure 4), which significantly outran those of the AC microcapsules.

\subsection{Oral Administration of Microcapsules on Gut Microflora} and Enzymatic Activities. The effect of microcapsules on human GI microflora was assessed by investigating the population of fecal marker microorganisms and the activity of 5 representative GI microbial enzymes in the simulated colonic media containing sterile microcapsules. Since the simulated GI model is a dynamic system, static experiments were performed in this study to maximize the effects. We found no marked differences in the tested microbial population including total aerobes, total anaerobes, Escherichia coli, Staphylococcus sp., and Lactobacillus sp. in the stimulated colonic media containing tested microcapsules of either AC or GCAC formulation when compared to the control media in the absence of tested microcapsules (Table 3 , all $P>.05$ ).

Figure 5 shows the effect of the microcapsules on the activities of microbial enzymes in the simulated transverse colon suspension. As time elapsed, a slight decline in the tested enzymatic activities was found in the simulated colonic fluids in the presence of microcapsules. As an exception, a decrease of more than $20 \%$ in the activity of $\beta$-glucuronidase was detected after 12 hours of contact with microcapsules, yet the loss remained at a similar level with extended incubation for up to 24 hours (Figure 5(c)). No significant differences in the alteration of enzymatic activities were found when the GCAC microcapsules were compared with the AC microcapsules $(P>.05)$.

\section{Discussion}

For successful exploitation of microcapsules as an oral delivery device, understanding of their performance under physiologically pertinent conditions that represent the human GI tract is essential. Although in vivo research using specific techniques such as histological sectioning [46], radiography [47] and gamma scintigraphy $[48,49]$ can progressively track the microcapsules in the GI tract, it remains difficult to follow the orally administered microcapsule at every stage of digestion on either animals or humans due to tedious processing, small size of the microcapsules, limited detection resolution and ethical constraints. In vitro simulation offers a number of advantages, for example, well-controlled experimental conditions and easy sampling, especially preferable for screening and examining a variety of samples. Buffered solutions, for example, with $\mathrm{pH}$ at $1-2$ or 6.5-7.5, are frequently reported in literature as the simulated GI fluids $[31,50,51]$; however they only represent the $\mathrm{pH}$ in the stomach and in the intestine, and do not mimic the complex human GI microbial ecosystem. While other ex vivo and in vitro simulated models including USP apparatus were also reported [52], most of them were static systems, where 


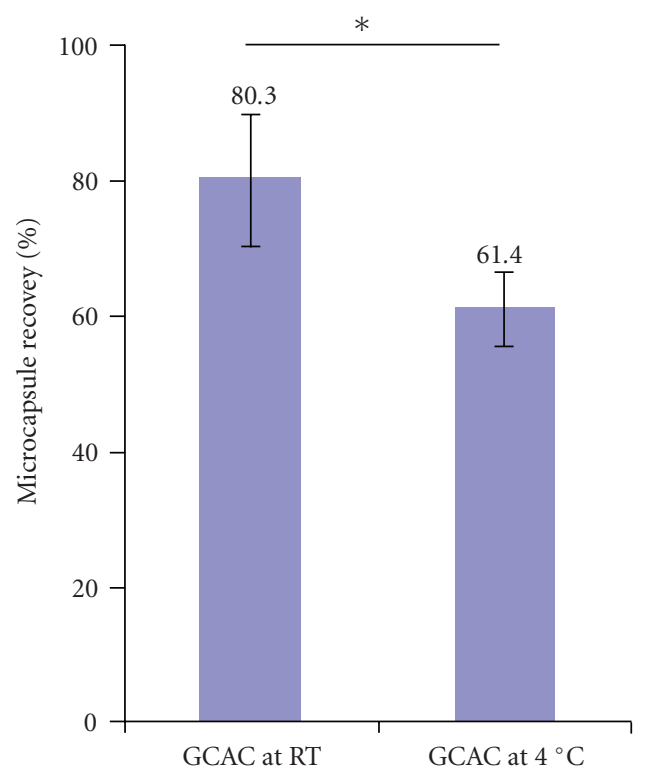

FIgURE 3: Recovery of microcapsules after 72 hours of the simulated human GI transit. * indicates significant difference at $P<.05$.

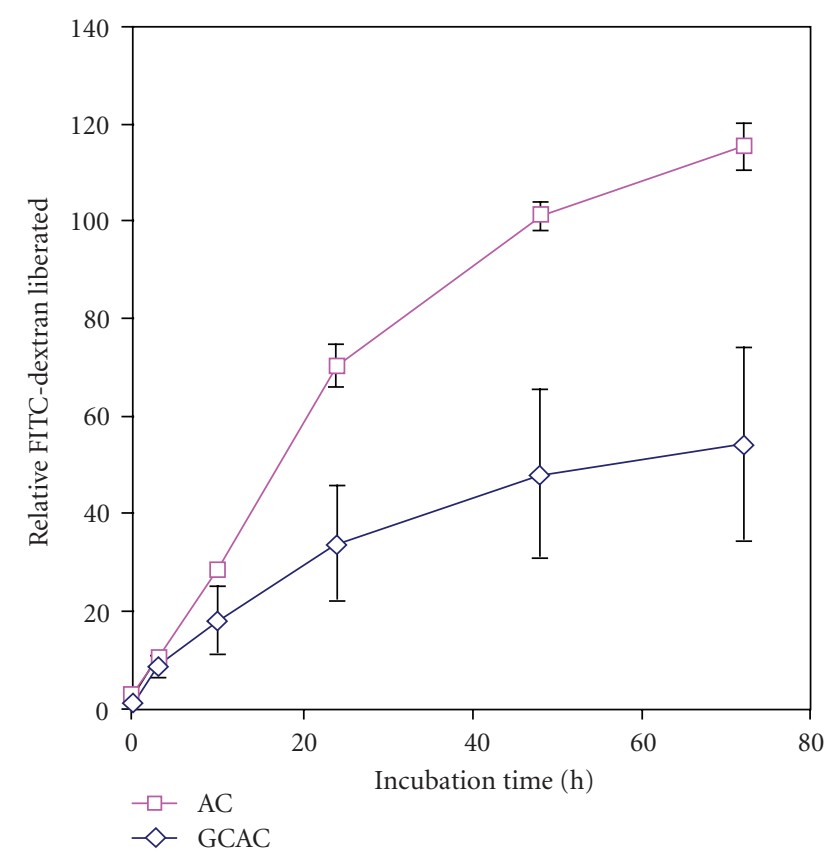

FIGURE 4: Leaching of the encapsulated FITC-dextran into the simulated intestinal medium following 1 hour simulated gastric exposure. Error bars indicate standard deviation of the mean $(n=$ $3)$.

fluids cannot be continuously transferred into the sequential GI compartments. Our present study used a dynamic computer-controlled human simulated GI model, which mimics the gradual transit of ingested materials through the simulated GI tract from feeding to discharge. It also maintains the microbial community actually presented in the human intestinal system, which allows for assessing the behavior of the microcapsules under more physiologically pertinent conditions.

As has been noted earlier, it is imperative that microcapsules maintain physical integrity during the GI transit to prevent the leaking of genetically engineered cells, which is strongly dependent on the microcapsule's robustness and stability. So far few studies were reported to address this matter [44]. The present study investigated the behaviour of the GCAC microcapsules in the simulated human GI environment. When exposed to the simulated GI media, the microcapsules experienced the simulated transit through the GI tract including $\mathrm{pH}$ fluctuation, enzymatic degradation, microorganism actions, mechanical stresses, as well as other related chemical and physiological constraints. The exposure time was chosen according to the maximum period retention in the human GI tract that represents the most challenging case for the tested microcapsules. The better-preserved morphology and higher retrieval rate associated with the GCAC microcapsules cross-linked at RT (Figures 2 and 3) indicated improved membrane stability by higher degree of cross-linking. Moreover, negligible amounts of encapsulated fluorescent probe were released into the incubation GI media from the GCAC microcapsules (Figure 4), suggesting that the integrity of the microcapsule membrane was withheld. In contrast, the non-cross-linked AC microcapsules were found vulnerable to structural disruption in the harsh GI condition, likely due to the relatively weak polyelectrolyte complexation of membrane materials that are prone to gastric and proteolytic degradation. These findings corroborated the results reported previously $[44,53-55]$. The present study demonstrated that covalent cross-linking of microcapsule membranes by genipin substantially improved the resistance to membrane degradation in the simulated human GI environment.

Another important prerequisite of oral therapy utilizing microencapsulation is that the administration of microcapsules should not disturb the natural colonic flora, particularly when prolonged and repeated oral intake of a rather large quantity of microcapsules is required for therapy. Microcapsules are made of various materials and chemicals through complexation and cross-linking reactions, all of which may have an effect on the biocompatibility of the final microcapsules. In particular, the well-balanced gut microbiota are important in maintaining human health [56, 57] and should not be altered by the intake of microcapsules. When taken into account the static nature and a rather large dosage ( $1.0 \mathrm{~g}$ of microcapsules in $10 \mathrm{~mL}$ of intestinal fluid) of the experiments in this in vitro study, our results suggested that the materials used to construct the microcapsules did not evoke appreciable adverse effects on the human intestinal flora and that genipin cross-linked chitosan membranes did not compromise the biocompatibility of the microcapsules when compared to the non-cross-linked subjects. Decrease in the activities of the tested microbial enzymes in the simulated colonic media containing microcapsules was detected. It could possibly be attributed to the binding effect or diffusion of the enzymes to the microcapsules, though further research may continue to elucidate the consequence. 


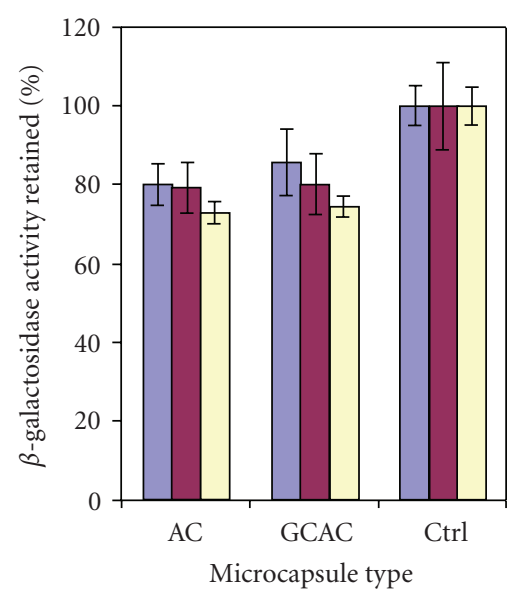

(a)

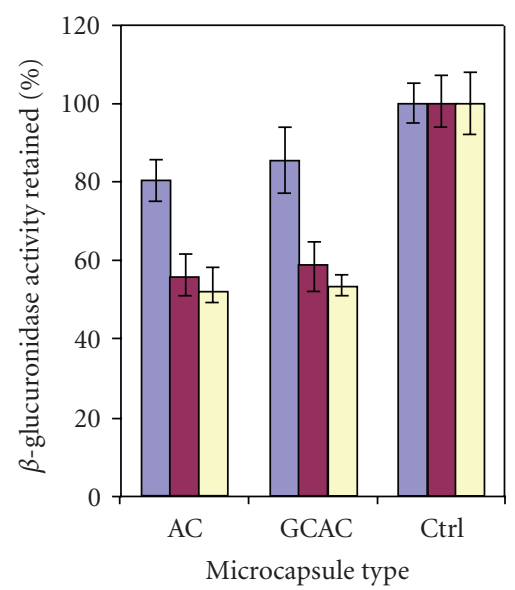

(c)

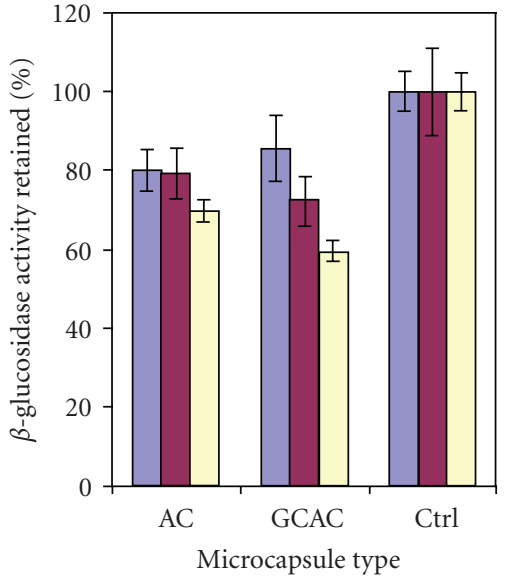

(b)

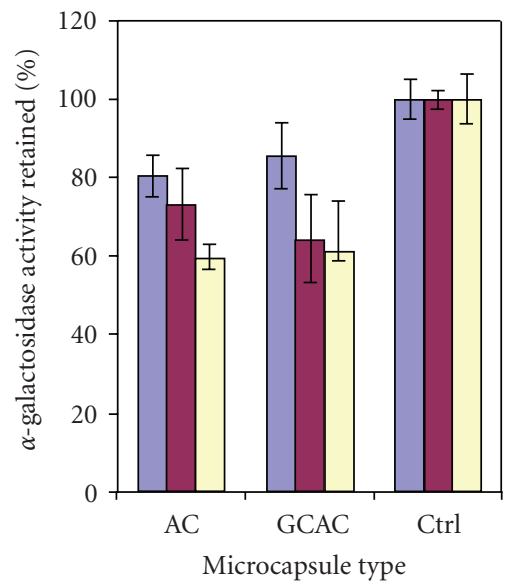

(d)

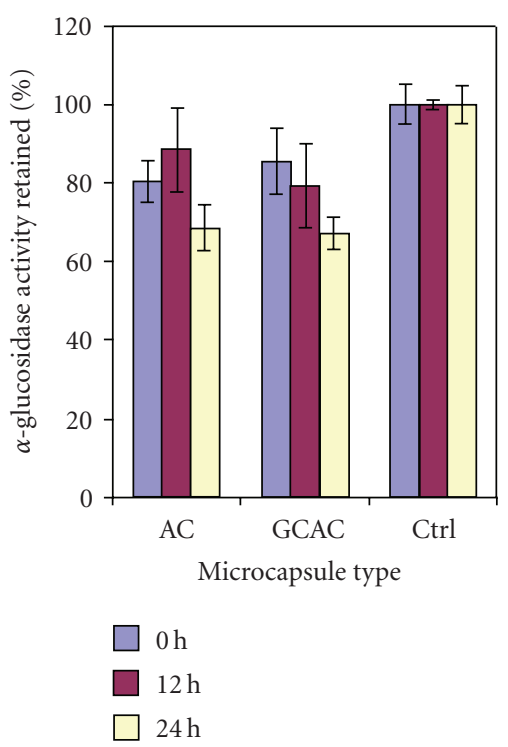

(e)

FIGURE 5: Percent microbial enzyme activities retained in the suspension of the simulated human transverse colon in the presence of microcapsules relative to that in the absence of microcapsules (control). Values for the controls at each time point were normalized to $100 \%$ and were used to calculate the percent enzyme activities retained in the microcapsule-containing media at corresponding time points. (a) $\beta$-galactosidase; (b) $\beta$-glucosidase; (c) $\beta$-glucuronidase; (d) $\alpha$-galactosidase; and (e) $\alpha$-glucosidase. 
TABLE 3: Effects of microcapsules on fecal marker microbes in the simulated transverse colon.

\begin{tabular}{|c|c|c|c|c|}
\hline \multirow{2}{*}{ Microbes } & \multirow{2}{*}{ Incubation time $(\mathrm{h})$} & \multicolumn{3}{|c|}{ 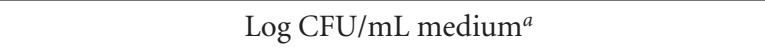 } \\
\hline & & $\mathrm{GCAC}^{b}$ & $\mathrm{AC}^{b}$ & Control $^{c}$ \\
\hline \multirow{4}{*}{ Total aerobes } & 0 & 8.41 & 8.41 & 8.41 \\
\hline & 6 & 8.37 & 8.32 & 8.36 \\
\hline & 12 & 8.36 & 8.37 & 8.39 \\
\hline & 24 & 8.22 & 8.17 & 8.04 \\
\hline \multirow{4}{*}{ Total anaerobes } & 0 & 8.44 & 8.44 & 8.44 \\
\hline & 6 & 8.37 & 8.33 & 8.41 \\
\hline & 12 & 8.37 & 8.43 & 8.40 \\
\hline & 24 & 8.41 & 8.30 & 8.03 \\
\hline \multirow{4}{*}{ Escherichia coli } & 0 & 8.31 & 8.31 & 8.31 \\
\hline & 6 & 8.18 & 7.97 & 8.18 \\
\hline & 12 & 8.20 & 8.02 & 8.41 \\
\hline & 24 & 8.10 & 8.03 & 8.68 \\
\hline \multirow{4}{*}{ Staphylococcus sp. } & 0 & 6.96 & 6.96 & 6.96 \\
\hline & 6 & 6.52 & 6.60 & 6.81 \\
\hline & 12 & 6.61 & 6.72 & 6.77 \\
\hline & 24 & 6.57 & 6.62 & 6.54 \\
\hline \multirow{4}{*}{ Lactobacillus sp. } & 0 & 5.48 & 5.48 & 5.48 \\
\hline & 6 & 5.45 & 5.52 & 5.51 \\
\hline & 12 & 5.35 & 5.40 & 5.32 \\
\hline & 24 & 5.50 & 5.46 & 5.37 \\
\hline
\end{tabular}

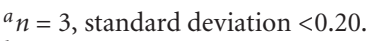

${ }^{b}$ Colonic suspension in the presence of microcapsules.

${ }^{c}$ Colonic suspension in the absence of microcapsules.

\section{Conclusions}

This study examined the performance of the genipin crosslinked alginate-chitosan microcapsules under physiologically pertinent GI conditions using a dynamic simulated human GI model. Results showed that these GCAC microcapsules possessed superior resistance against disintegration in the simulated GI environment and did not appreciably alter the normal gut flora. This novel microcapsule formulation is promising for oral delivery of genetically engineered bacteria; however, further studies on encapsulated cell viability in the gut lumen are needed before its full potential can be realized.

\section{Acknowledgments}

This paper was supported by the Canadian Institutes of Health Research (CIHR) MOP 64308. Postgraduate fellowships from the Natural Sciences and Engineering Research Council of Canada (to H. Chen and C. Martoni) and Fonds Québécois de la Recherche sur la Nature et les Technologies (to H. Chen and W. Ouyang) are greatly acknowledged. The authors also thank M. L. Jones for his help with the GI model set-up, T. Haque, M. Shafiei, and H. Wightman for assistance in the experiments.

\section{References}

[1] S. Prakash and J. Bhathena, "Live bacterial cells as orally delivered therapeutics," Expert Opinion on Biological Therapy, vol. 5, no. 10, pp. 1281-1301, 2005.

[2] L. Steidler, "Genetically engineered probiotics," Bailliere's Best Practice and Research in Clinical Gastroenterology, vol. 17, no. 5, pp. 861-876, 2003.

[3] M. Goldberg and I. Gomez-Orellana, "Challenges for the oral delivery of macromolecules," Nature Reviews Drug Discovery, vol. 2, no. 4, pp. 289-295, 2003.

[4] G. Orive, R. M. Hernández, A. Rodríguez Gascón et al., "History, challenges and perspectives of cell microencapsulation," Trends in Biotechnology, vol. 22, no. 2, pp. 87-92, 2004.

[5] G. Bergers and D. Hanahan, "Cell factories for fighting cancer," Nature Biotechnology, vol. 19, no. 1, pp. 20-21, 2001.

[6] J. Bloch, A. C. Bachoud-Lévi, N. Déglon et al., "Neuroprotective gene therapy for Huntington's disease, using polymer-encapsulated cells engineered to secrete human ciliary neurotrophic factor: results of a phase I study," Human Gene Therapy, vol. 15, no. 10, pp. 968-975, 2004.

[7] T. M. S. Chang, "Therapeutic applications of polymeric artificial cells," Nature Reviews Drug Discovery, vol. 4, no. 3, pp. 221-235, 2005. 
[8] M. L. Jones, H. Chen, W. Ouyang, T. Metz, and S. Prakash, "Microencapsulated genetically engineered Lactobacillus plantarum 80 ( $\mathrm{pCBH} 1)$ for bile acid deconjugation and its implication in lowering cholesterol," Journal of Biomedicine and Biotechnology, vol. 2004, no. 1, pp. 61-69, 2004.

[9] S. Prakash and T. M. S. Chang, "Microencapsulated genetically engineered live E. coli DH5 cells administered orally to maintain normal plasma urea level in uremic rats," Nature Medicine, vol. 2, no. 8, pp. 883-887, 1996.

[10] T.-A. Read, D. R. Sorensen, R. Mahesparan et al., "Local endostatin treatment of gliomas administered by microencapsulated producer cells," Nature Biotechnology, vol. 19, no. 1, pp. 29-34, 2001.

[11] C. J. D. Ross, L. Bastedo, S. A. Maier, M. S. Sands, and P. L. Chang, "Treatment of a lysosomal storage disease, mucopolysaccharidosis VII, with microencapsulated recombinant cells," Human Gene Therapy, vol. 11, no. 15, pp. 2117-2127, 2000.

[12] A. D. Tagalakis, I. A. Diakonov, I. R. Graham et al., "Apolipoprotein E delivery by peritoneal implantation of encapsulated recombinant cells improves the hyperlipidaemic profile in apoE-deficient mice," Biochimica et Biophysica Acta, vol. 1686, no. 3, pp. 190-199, 2005.

[13] J. M. Van Raamsdonk, C. J. D. Ross, M. A. Potter et al., "Treatment of hemophilia B in mice with nonautologous somatic gene therapeutics," Journal of Laboratory and Clinical Medicine, vol. 139, no. 1, pp. 35-42, 2002.

[14] L. R. Johnson, Physiology of the Gastrointestinal Tract, Elsevier Academic Press, Burlington, Mass, USA, 2006.

[15] J. Chin, B. Turner, I. Barchia, and A. Mullbacher, "Immune response to orally consumed antigens and probiotic bacteria," Immunology and Cell Biology, vol. 78, no. 1, pp. 55-66, 2000.

[16] N. Ishibashi and S. Yamazaki, "Probiotics and safety," American Journal of Clinical Nutrition, vol. 73, no. 2, pp. 465S-470S, 2001.

[17] C. P. Champagne, N. J. Gardner, and D. Roy, "Challenges in the addition of probiotic cultures to foods," Critical Reviews in Food Science and Nutrition, vol. 45, no. 1, pp. 61-84, 2005.

[18] S. Salminen, A. von Wright, L. Morelli et al., "Demonstration of safety of probiotics - a review," International Journal of Food Microbiology, vol. 44, no. 1-2, pp. 93-106, 1998.

[19] S. Freiberg and X. X. Zhu, "Polymer microspheres for controlled drug release," International Journal of Pharmaceutics, vol. 282, no. 1-2, pp. 1-18, 2004.

[20] L. R. Moses, K. J. Dileep, and C. P. Sharma, "Beta cyclodextrininsulin-encapsulated chitosan/alginate matrix: oral delivery system," Journal of Applied Polymer Science, vol. 75, no. 9, pp. 1089-1096, 2000.

[21] E. Nechaeva, "Development of oral microencapsulated forms for delivering viral vaccines," Expert Review of Vaccines, vol. 1, no. 3, pp. 385-397, 2002.

[22] G. Orive, A. R. Gascón, R. M. Hernández, A. Domínguez-Gil, and J. L. Pedraz, "Techniques: new approaches to the delivery of biopharmaceuticals," Trends in Pharmacological Sciences, vol. 25, no. 7, pp. 382-387, 2004.

[23] V. R. Sinha and A. Trehan, "Biodegradable microspheres for protein delivery," Journal of Controlled Release, vol. 90, no. 3, pp. 261-280, 2003.

[24] A. Bartkowiak, "Optimal conditions of transplantable binary polyelectrolyte microcapsules," Annals of the New York Academy of Sciences, vol. 944, pp. 120-134, 2001.

[25] D. W. Green, I. Leveque, D. Walsh et al., "Biomineralized polysaccharide capsules for encapsulation, organization, and delivery of human cell types and growth factors," Advanced Functional Materials, vol. 15, no. 6, pp. 917-923, 2005.
[26] T. Haque, H. Chen, W. Ouyang et al., "In vitro study of alginate-chitosan microcapsules: an alternative to liver cell transplants for the treatment of liver failure," Biotechnology Letters, vol. 27, no. 5, pp. 317-322, 2005.

[27] D. Maysinger, O. Berezovskaya, and S. Fedoroff, "The hematopoietic cytokine colony stimulating factor 1 is also a growth factor in the CNS.2. Microencapsulated CSF-1 and LM-10 cells as delivery systems," Experimental Neurology, vol. 141, no. 1, pp. 47-56, 1996.

[28] D. Maysinger, K. Krieglstein, J. Filipovic-Grcic, M. Sendtner, K. Unsicker, and P. Richardson, "Microencapsulated ciliary neurotrophic factor: physical properties and biological activities," Experimental Neurology, vol. 138, no. 2, pp. 177-188, 1996.

[29] T. Chandy, D. L. Mooradian, and G. H. R. Rao, "Evaluation of modified alginate-chitosan-polyethylene glycol microcapsules for cell encapsulation," Artificial Organs, vol. 23, no. 10, pp. 894-903, 1999.

[30] G. Klinkenberg, K. Q. Lystad, D. W. Levine, and N. Dyrset, "Cell release from alginate immobilized Lactococcus lactis ssp. lactis in chitosan and alginate coated beads," Journal of Dairy Science, vol. 84, no. 5, pp. 1118-1127, 2001.

[31] W. Krasaekoopt, B. Bhandari, and H. Deeth, "The influence of coating materials on some properties of alginate beads and survivability of microencapsulated probiotic bacteria," International Dairy Journal, vol. 14, no. 8, pp. 737-743, 2004.

[32] G. Orive, A. Bartkowiak, S. Lisiecki et al., "Biocompatible oligochitosans as cationic modifiers of alginate/Ca microcapsules," Journal of Biomedical Materials ResearchPart B, vol. 74, no. 1, pp. 429-439, 2005.

[33] A. Zanina, A. Vilesov, and T. Budtova, "Shear-induced solvent release from gel particles: application to drug-delivery systems," International Journal of Pharmaceutics, vol. 242, no. 1-2, pp. 137-146, 2002.

[34] C. Djerassi, J. D. Gray, and F. A. Kincl, "Naturally occurring oxygen heterocyclics. 9. Isolation and characterization of genipin," Journal of Organic Chemistry, vol. 25, no. 12, pp. 2174-2177, 1960.

[35] J.-E. Park, J.-Y. Lee, H.-G. Kim, T.-R. Hahn, and Y.-S. Paik, "Isolation and characterization of water-soluble intermediates of blue pigments transformed from geniposide of Gardenia jasminoides," Journal of Agricultural and Food Chemistry, vol. 50, no. 22, pp. 6511-6514, 2002.

[36] H. Chen, W. Ouyang, M. Jones et al., "Preparation and characterization of novel polymeric microcapsules for live cell encapsulation and therapy," Cell Biochemistry and Biophysics, vol. 47, no. 1, pp. 159-167, 2007.

[37] J. Jin, M. Song, and D. J. Hourston, "Novel chitosan-based films cross-linked by genipin with improved physical properties," Biomacromolecules, vol. 5, no. 1, pp. 162-168, 2004.

[38] F.-L. Mi, Y.-C. Tan, H.-F. Liang, and H.-W. Sung, "In vivo biocompatibility and degradability of a novel injectable-chitosanbased implant," Biomaterials, vol. 23, no. 1, pp. 181-191, 2002.

[39] H. Chen, W. Ouyang, B. Lawuyi, and S. Prakash, "Genipin cross-linked alginate-chitosan microcapsules: membrane characterization and optimization of cross-linking reaction," Biomacromolecules, vol. 7, no. 7, pp. 2091-2098, 2006.

[40] H. Chen, W. Ouyang, C. Martoni, and S. Prakash, "Genipin cross-linked polymeric alginate-chitosan microcapsules for oral delivery: in-vitro analysis," International Journal of Polymer Science, vol. 2009, Article ID 617184, 16 pages, 2009. 
[41] K. Molly, M. V. Woestyne, and W. Verstraete, "Development of a 5-step multi-chamber reactor as a simulation of the human intestinal microbial ecosystem," Applied Microbiology and Biotechnology, vol. 39, no. 2, pp. 254-258, 1993.

[42] N. Berrada, J. F. Lemeland, G. Laroche, P. Thouvenot, and M. Piaia, "Bifidobacterium from fermented milks: survival during gastric transit," Journal of Dairy Science, vol. 74, no. 2, pp. 409-413, 1991.

[43] J. O. Berg, C. E. Nord, and T. Wadstrom, "Formation of glycosidases in batch and continuous culture of Bacteroides fragilis," Applied and Environmental Microbiology, vol. 35, no. 2, pp. 269-273, 1978.

[44] H. Chen, W. Ouyang, M. Jones, T. Haque, B. Lawuyi, and S. Prakash, "In-vitro analysis of APA microcapsules for oral delivery of live bacterial cells," Journal of Microencapsulation, vol. 22, no. 5, pp. 539-547, 2005.

[45] F. A. Leblond, J. Tessier, and J.-P. Hallé, "Quantitative method for the evaluation of biomicrocapsule resistance to mechanical stress," Biomaterials, vol. 17, no. 21, pp. 2097-2102, 1996.

[46] J. Kovacs-Nolan and Y. Mine, "Microencapsulation for the gastric passage and controlled intestinal release of immunoglobulin Y," Journal of Immunological Methods, vol. 296, no. 1-2, pp. 199-209, 2005.

[47] R. Narayani and K. P. Rao, "Polymer-coated gelatin capsules as oral delivery devices and their gastrointestinal tract behaviour in humans," Journal of Biomaterials Science. Polymer Edition, vol. 7, no. 1, pp. 39-48, 1995.

[48] Y. Sato, Y. Kawashima, H. Takeuchi, H. Yamamoto, and Y. Fujibayashi, "Pharmacoscintigraphic evaluation of riboflavincontaining microballoons for a floating controlled drug delivery system in healthy humans," Journal of Controlled Release, vol. 98, no. 1, pp. 75-85, 2004.

[49] I. R. Wilding, D. Clark, H. Wray, J. Alderman, N. Muirhead, and C. R. Sikes, "In vivo disintegration profile of encapsulated and nonencapsulated sumatriptan: gamma scintigraphy in healthy volunteers," Journal of Clinical Pharmacology, vol. 45, no. 1, pp. 101-105, 2005.

[50] A. Lamprecht, H. Yamamoto, H. Takeuchi, and Y. Kawashima, "Design of $\mathrm{pH}$-sensitive microspheres for the colonic delivery of the immunosuppressive drug tacrolimus," European Journal of Pharmaceutics and Biopharmaceutics, vol. 58, no. 1, pp. 37-43, 2004.

[51] T. Reddy and S. Tammishetti, "Gastric resistant microbeads of metal ion cross-linked carboxymethyl guar gum for oral drug delivery," Journal of Microencapsulation, vol. 19, no. 3, pp. 311-318, 2002.

[52] F. Delie, "Evaluation of nano- and microparticle uptake by the gastrointestinal tract," Advanced Drug Delivery Reviews, vol. 34, no. 2-3, pp. 221-233, 1998.

[53] S. R. Bhatia, S. F. Khattak, and S. C. Roberts, "Polyelectrolytes for cell encapsulation," Current Opinion in Colloid and Interface Science, vol. 10, no. 1-2, pp. 45-51, 2005.

[54] B. R. S. Hsu, S. H. Fu, J. S. Tsai, Y. Y. Huang, H. S. Huang, and K. S. S. Chang, "The plasminogen-plasmin fibrinolytic system accelerates degradation of alginate-poly-L-lysine-alginate microcapsules in vitro," Transplantation Proceedings, vol. 29, no. 3, pp. 1877-1880, 1997.

[55] H. Zimmermann, D. Zimmermann, R. Reuss et al., "Towards a medically approved technology for alginate-based microcapsules allowing long-term immunoisolated transplantation," Journal of Materials Science, vol. 16, no. 6, pp. 491-501, 2005.
[56] K. Hirayama and J. Rafter, "The role of lactic acid bacteria in colon cancer prevention: mechanistic considerations," Antonie Van Leeuwenhoek, International Journal of General and Molecular Microbiology, vol. 76, no. 1-4, pp. 391-394, 1999.

[57] G. L. Simon and S. L. Gorbach, "Intestinal flora in health and disease," Gastroenterology, vol. 86, no. 1, pp. 174-193, 1984. 

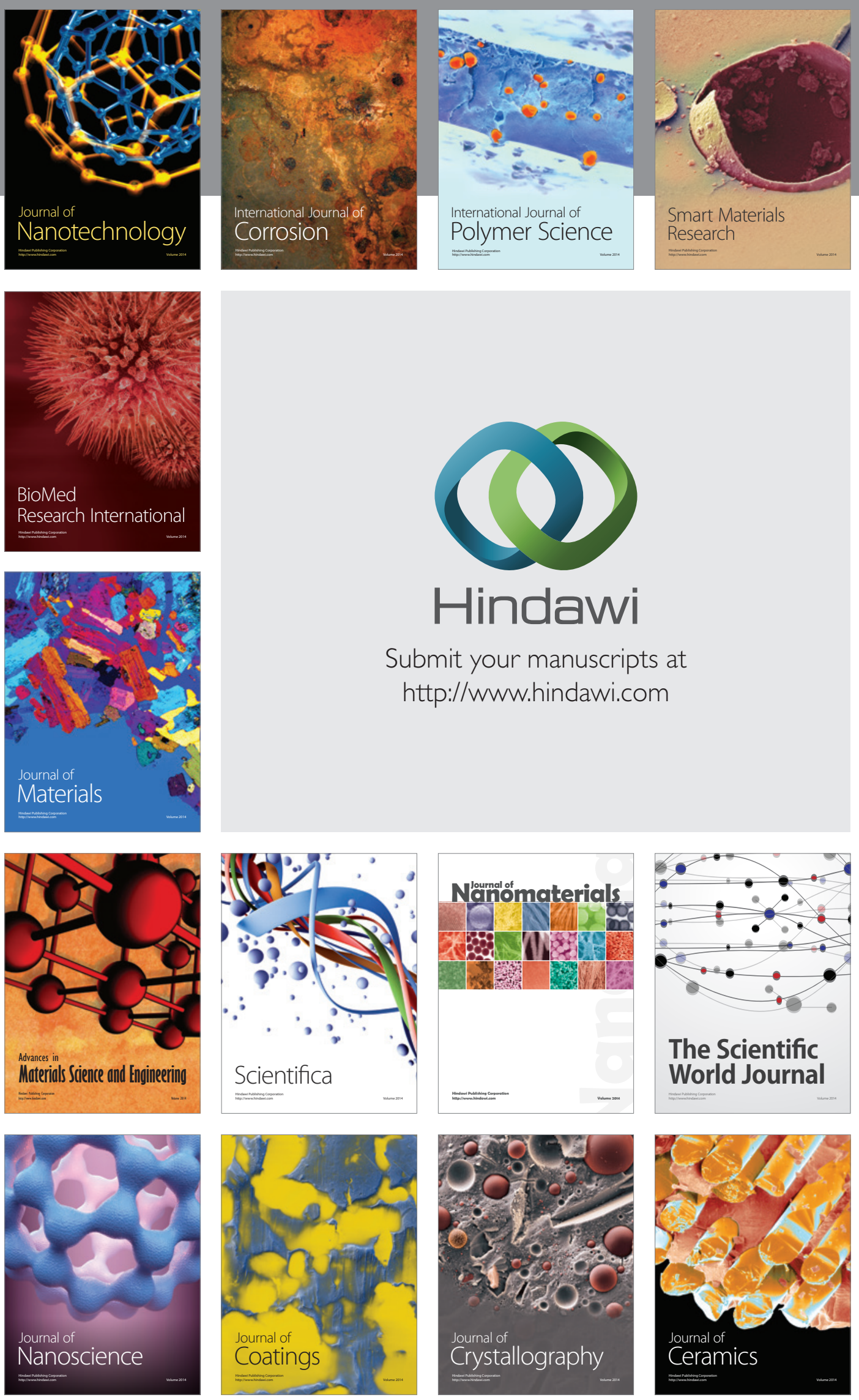

The Scientific World Journal

Submit your manuscripts at

http://www.hindawi.com

\section{World Journal}

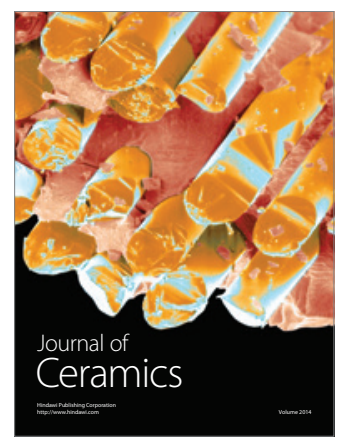

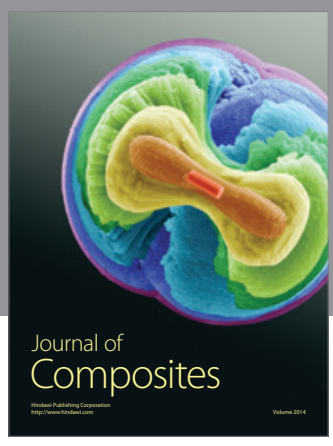
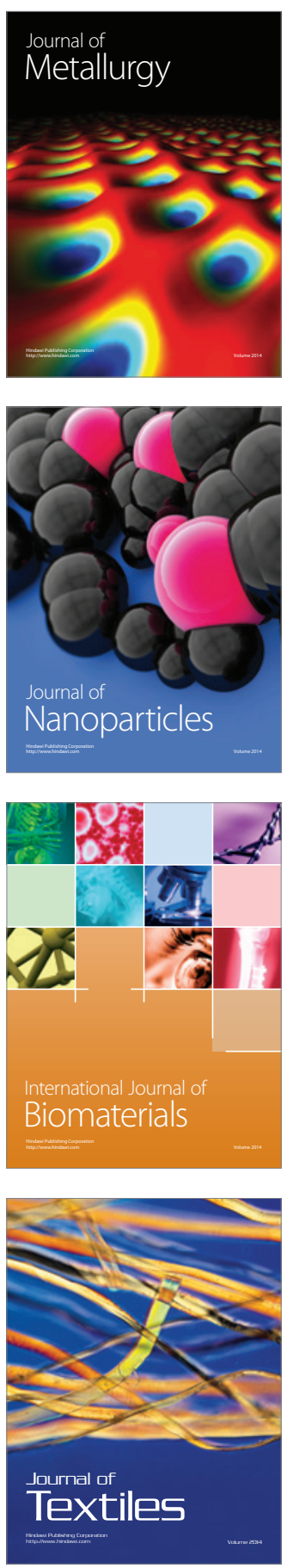